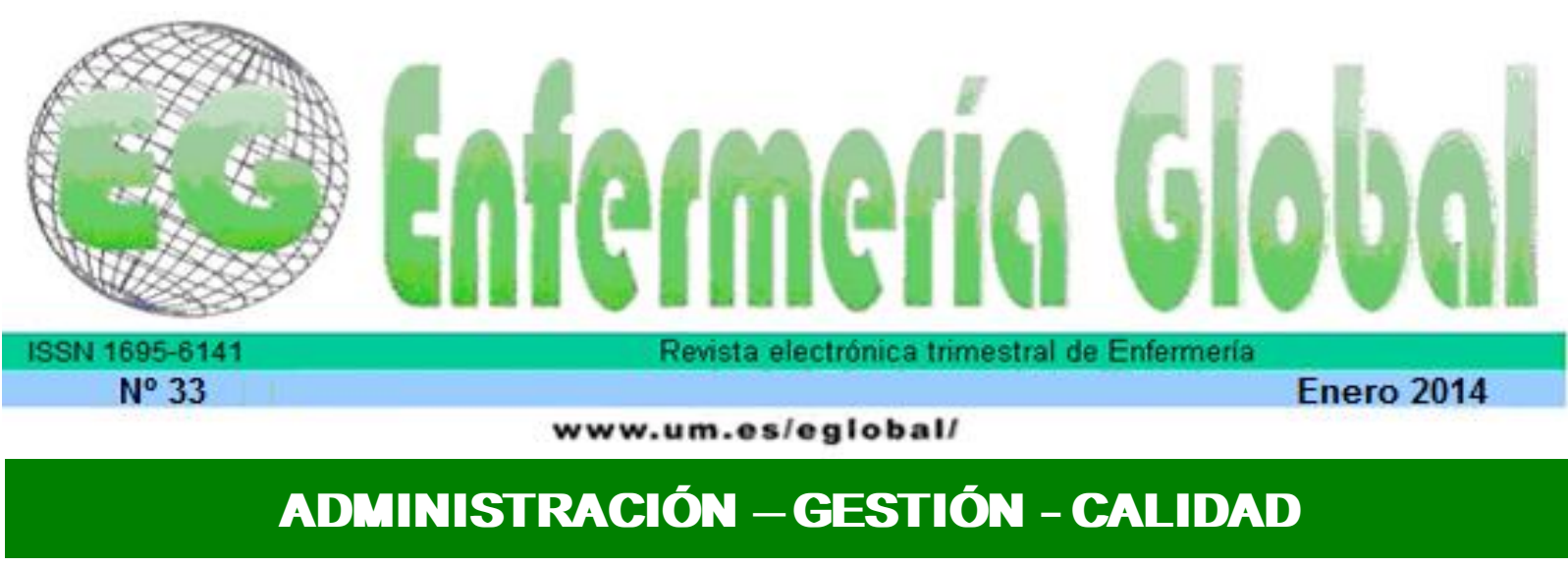

\title{
Experiencia de un sistema de reporte de errores de medicación en un hospital docente
}

Adverse drug event reporting system: a university hospital experience

\author{
${ }^{*}$ Castro Lara, Ariel ${ }^{* *}$ Sotomayor Nieto, Julia ${ }^{* *}$ Sepúlveda Vargas, Yasna \\ ${ }^{* *}$ Mena Velasquez, Sandra \\ *Farmacéutico, MSc. Epidemiología Clínica E-mail: acastro@hcuch.cl "**Enfermera Universitaria. \\ Hospital Clínico Universidad de Chile.
}

Palabras clave: Errores de Medicación; Eventos Adversos de Medicamentos; Reporte. Keywords: Medication Error; Adverse Drug Events; Reports.

\section{RESUMEN}

Introducción: Los errores de medicación (EM) tienen un impacto en la morbi-mortalidad de los pacientes, como así también consecuencias económicas para el individuo, los sistemas de salud y la sociedad. Una forma de identificarlos es a través del sistema de reportes.

Objetivo: Presentar la experiencia de uso de reportes en un hospital docente universitario.

Metodología: Análisis descriptivo de reportes de EM. Se utilizaron como fuentes de información el sistema de censo y los reportes de los errores en la medicación.

Resultados: La tasa de reportes de EM fue de $1.2 \times 1000$ pacientes. Los reportes principalmente provinieron de servicios de tipo médicos (39\%) y de Unidades de Pacientes Críticos-Aislamiento (34\%). Los EM más frecuentes estuvieron en la administración (47\%) y dispensación (27\%). El 69\% los errores notificados llegaron al paciente, necesitándose algún tipo de intervención en el $68 \%$ de esos casos. La gravedad de los EM fue importante en el $47 \%$ de los casos, pudiéndose prevenir en el $97 \%$.

Conclusiones: Los EM son una realidad presente en los centros asistenciales, que se puede prevenir. El sistema de reportes de EM es una herramienta útil en la identificación de sus causas.

\section{ABSTRACT}

Introduction: The medication error (ME) has an impact in the morbi-mortality of the patients, as this way also the economic consequences for the individual, the systems of health and the society. A way of identifying them is across the system of reports. The aim of this communication is to present the experience of use of reports in an teaching hospital. 
Methodology: Descriptive Analysis of ME reports. There were in use as sources of information the system of census and the reports of the ME.

Results: The rate of ME's reports was of $1.2 \times 1000$ patients. The reports principally came from medical services (39\%) and 34\% from Intensive Care Units $34 \%$. More frequent MM was in the administration $(47 \%)$ and dispensation (27\%). The notified ME $69 \%$ came to the patient, some type of intervention being needed in $68 \%$ of these cases. The gravity of ME was important in $47 \%$ of the cases, being able to preventable $97 \%$.

Conclusions: ME is a present reality in our hospital, which it is possible to anticipate. The system of ME reports is a useful tool in the identification that causes root.

\section{INTRODUCCIÓN}

Las muertes atribuibles a errores médicos, entre los cuales se encuentran los errores en la medicación (EM), exceden según el Instituto de Medicina (IOM) de EEUU a los Accidentes Automovilísticos, Cáncer de Mama y SIDA (1). Estos EM, se deben principalmente a que el Sistema de Utilización de Medicamentos cada vez se hace más complejo, constituyéndose como el principal factor de EM, el que se inicia con el proceso de selección de un arsenal fármaco terapéutico en diferentes niveles (gubernamental, provincial, hospitalario), posteriormente el acto de la prescripción por parte de los profesionales del equipo de salud, principalmente médicos, y luego la dispensación o preparación del medicamento por la unidad de farmacia para que sea administrado por el personal de enfermería. El proceso termina con la monitorización de la respuesta clínica del paciente a la prescripción realizada y de acuerdo a ello se mantiene o modifica la terapia, partiendo nuevamente el proceso.

La incidencia de EM en hospitales se encuentran entre 11.5 x 1000 pacientes día y $6.2 \times 100$ hospitalizaciones ${ }^{(2,3)}$.

Puede afirmarse con seguridad que los acontecimientos adversos relacionados con los EM conllevan el descenso significativo de la calidad de vida del paciente, en ocasiones por deterioro orgánico y/o discapacidades físicas e incluso la muerte ${ }^{(4)}$. Las consecuencias económicas que se derivan de los EM se deben fundamentalmente a indemnizaciones por mala práctica, prolongación de la estancia media hospitalaria, necesidad de asistencia sanitaria paliativa y pérdida de productividad, invalidez o muerte ${ }^{(5)}$.

Desde el punto de vista social, se muestra una intolerancia creciente a los errores en los cuidados sanitarios. La repercusión social de los errores de medicación debe estudiarse desde una doble vertiente, por un lado, desde el punto de vista de los pacientes y sus familiares o cuidadores, puede afirmarse que ser testigo o sufrir un EM compromete la confianza en el Sistema Sanitario; y por otro lado, si tenemos en cuenta a los profesionales sanitarios que han cometido un error, vemos cómo el ser conscientes de ello les conduce a desconfiar de sí mismos en lo sucesivo, a la desmotivación y miedo a cometer errores ${ }^{(6)}$.

Debido a la connotación sanitaria, económica y social de los EM, se han implementado diversas acciones de manera de disminuir al máximo esta situación, entre las cuales están la prescripción electrónica (7), equipo de enfermería específicamente dedicado la administración de medicamentos ${ }^{(8)}$, sistemas de dispensación en dosis unitarias ${ }^{(9)}$, entre otras. Sin embargo, la implementación de sistemas seguros de uso de medicamento no asegura por si sólo el éxito, para ello se 
hace necesario la evaluación de las medidas implementadas. Algunas metodologías utilizadas para la evaluación y detección de EM que han sido referidas en literatura se encuentra la revisión de historias clínicas (10), la observación directa de los procesos $^{(11)}$, monitorización de parámetros de laboratorios indicativos de un EM ${ }^{(12)}$, etc.

Sin embargo, la implementación de sistemas seguros de uso de medicamentos no asegura por si sólo el éxito; para ello, se hace necesario la evaluación de las medidas implementadas. Entre algunas metodologías utilizadas para la evaluación y detección de EM que han sido reportadas en literatura se encuentran: la revisión de historias clínicas ${ }^{(10)}$, la observación directa de los procesos ${ }^{(11)}$ y el reporte de EM ${ }^{(13)}$.

El objetivo del presente trabajo es dar a conocer la experiencia obtenida en dos años de aplicación de los reportes de EM.

\section{METODOLOGÍA}

El estudio se realizó en un hospital universitario que cuenta con 600 camas, todas las especialidades médicas, el cual tiene anualmente alrededor de 21 mil egresos hospitalarios.

La información se obtuvo del reporte de incidentes de enfermería, el cual contiene una sección acerca de la medicación en que se registra: el tipo de incidente ocurrido, el o los medicamentos involucrados, el servicio, la hora y las medidas tomadas para la resolución del incidente. Además, desde el sistema de censo del hospital se obtuvieron datos demográficos generales como edad y sexo de los pacientes, días de hospitalización, y datos de morbilidad según la Clasificación Internacional de Enfermedades $\mathrm{ClE}-10^{(14)}$. Los medicamentos involucrado se clasificaron según la Clasificación Anatomo-Terapéutica de la OMS ${ }^{(15)}$.

Los EM se clasificaron de acuerdo al proceso en el que se inició el error (prescripción, dispensación, preparación, dispensación) y luego se categorizaron de acuerdo a la gravedad según el National Coordinating Council for Medication Error Reporting and Prevention (NCCMERP) ${ }^{(16)}$. Los datos recolectados se ingresaron a una planilla MSExcel®, para su análisis y clasificación.

\section{RESULTADOS}

Durante los dos años en los cuales se revisaron los reportes, se cuantificaron $59 \mathrm{EM}$, lo que corresponde a 1.19 reportes $\times 1000$ pacientes. Los cuales se generaron principalmente desde Servicios de tipo Médico y de las Unidades de Pacientes Críticos y de Aislamiento (Tabla 1). 
Tabla 1 Distribución del Origen de los Reportes de EM

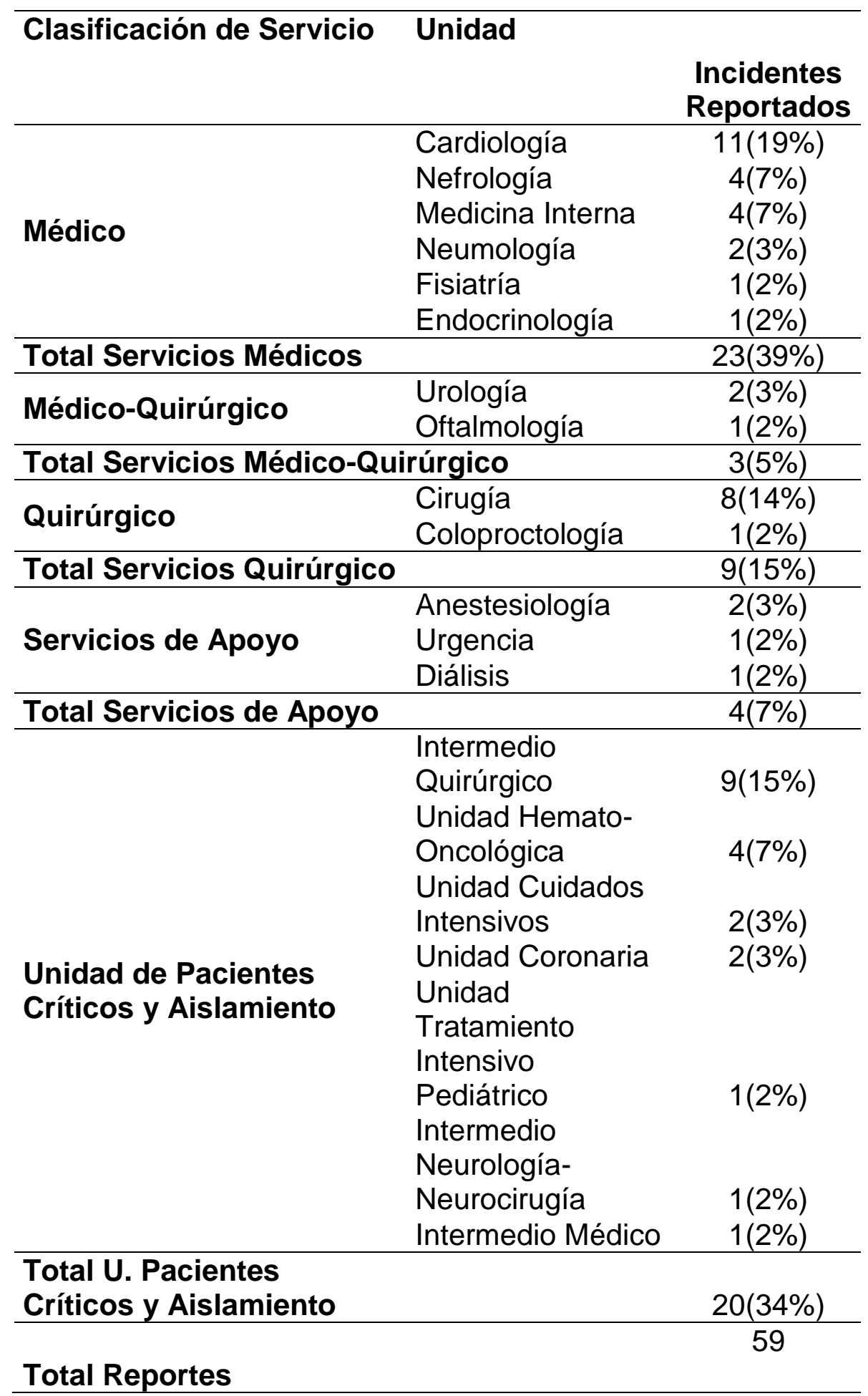

Los pacientes que estuvieron involucrados en un reporte de EM, fueron mayoritariamente mujeres (58\%), con una edad promedio de 55 años y un promedio de 17 días de hospitalización. Las patologías más frecuentes según CIE-10 fueron relacionadas al Sistema Circulatorio (31\%) y Neoplasmas (25\%) (Tabla 2). 
Tabla 2.- Características Demográficas y de Morbilidad de los Pacientes Involucrados en EM.

\begin{tabular}{lr}
\hline Sexo & $\begin{array}{l}\text { Mujer 34 } \\
(58 \%) \\
\text { Hombre 45 } \\
(42 \%)\end{array}$ \\
Edad (años) & $55 \pm 21$ \\
Días Hospitalización & $17 \pm 19$ \\
\hline \multicolumn{2}{c}{ Clasificación Diagnóstica Principal } \\
\hline Enf. Sist. Circulatorio & $18(31 \%)$ \\
Neoplasmas & $15(25 \%)$ \\
Enf. Sist. Genito-Urinario & $7(12 \%)$ \\
Enf. Sist. Digestivo & $7(12 \%)$ \\
Enf. Sist. Respiratorio & $3(5 \%)$ \\
Enf. Sist. Nervioso Central & $2(3 \%)$ \\
Enf. Endocrinas & $2(3 \%)$ \\
Enf. Embarazo, Parto y & $1(2 \%)$ \\
Puerperio & \\
Enf. Infecciosas y & $1(2 \%)$ \\
Parasitarias & $1(2 \%)$ \\
Enf. de la Piel & $1(2 \%)$ \\
Enf. del Aparato Locomotor & $1(2 \%)$ \\
Lesiones, heridas, & \\
intoxicaciones y otros & \\
factores externos & \\
\hline
\end{tabular}

Los medicamentos involucrados en EM según la clasificación ATC, pertenecían en un $19 \%$ al Sistema Hematopoyéctico (Heparina (5\%), Acenocumarol (3\%)), y en un 15\% al grupo de Antiinfecciosos de Uso Sistémico (Vancomicina, 3\%). (Tabla 3). Es importante señalar que la mayor parte de los medicamentos involucrados eran para administración endovenosa.

Tabla 3 Descripción de los reportes de EM según Grupos Terapéuticos

\begin{tabular}{|c|c|c|}
\hline Grupo Terapéutico & Med Involucrado & $\begin{array}{l}\text { Frecuencia } \\
\text { de } \\
\text { Incidentes } \\
\text { Reportados }\end{array}$ \\
\hline \multirow{6}{*}{$\begin{array}{l}\text { Ag. Antineoplásicos e } \\
\text { Inmunoduladores }\end{array}$} & Adriamicina & $1(2 \%)$ \\
\hline & Aracnósido C & $1(2 \%)$ \\
\hline & Ciclofosfamida & $1(2 \%)$ \\
\hline & Ciclosporina & $1(2 \%)$ \\
\hline & Cetuximab & $1(2 \%)$ \\
\hline & Etopósido & $2(3 \%)$ \\
\hline \multicolumn{2}{|c|}{ Total Ag. Antineoplásicos e Inmunomoduladores } & $7(12 \%)$ \\
\hline \multirow{4}{*}{$\begin{array}{l}\text { Med. Antiinfecciosos de Uso } \\
\text { Sistémico }\end{array}$} & Ampicilina & $1(2 \%)$ \\
\hline & Anfotericina B & $1(2 \%)$ \\
\hline & Ciprofloxacino & $1(2 \%)$ \\
\hline & Cloxacilina & $1(2 \%)$ \\
\hline
\end{tabular}


Total Med. Antiinfecciosos de Uso Sistémico

Med. Hormonales Sistémicos

Total Med. Hormonales Sistémicos

Med. Sistema Cardiovascular

Total Med. Sistema Cardiovascular

Med Sistema Nervioso

Total Med. Sistema Nervioso

\begin{tabular}{llr} 
Total Med. Sistema Nervioso & \multicolumn{1}{c}{$\begin{array}{c}8(14 \%) \\
\text { Grupo Terapéutico }\end{array}$} & $\begin{array}{l}\text { Frecuencia } \\
\text { de Incidentes } \\
\text { Reportados }\end{array}$ \\
\hline & Med Involucrado & $2(3 \%)$ \\
& Aminoácidos & $1(2 \%)$ \\
Med. Sistema Hematopoyético & Bicarbonato de Sodio & $1(2 \%)$ \\
& Calcio & $1(2 \%)$ \\
& Enoxiparina & $2(3 \%)$ \\
& Heparina & $3(5 \%)$ \\
\hline Total Med. Sistema Hematopoyético & KCl & $1(2 \%)$ \\
\hline \multirow{2}{*}{ Med. Sistema Músculo Esquelético } & Ciclobenzaprina & $11(19 \%)$ \\
& Dipirona & $1(2 \%)$ \\
& Ketoprofeno & $2(3 \%)$ \\
\hline Total Med. Sistema Músculo & & $1(2 \%)$ \\
\hline Esquelético & & $4(7 \%)$ \\
Med. Sistema Respiratorio & Epinefrina Racémica & $1(2 \%)$ \\
\hline Total Med. Sistema Respiratorio & Noradrenalina & $1(2 \%)$ \\
\hline & & $2(3 \%)$ \\
\hline Med. Tracto Alimentario y & Glibenclamida & $1(2 \%)$ \\
Metabolismo & Hidrocortisona & $1(2 \%)$ \\
& Insulina Cristalina & $1(2 \%)$ \\
& Insulina NPH & $2(3 \%)$ \\
\hline Total Med. Tracto Alimentario y & Nutrición Parenteral & $1(2 \%)$ \\
Metabolismo & Total & $1(2 \%)$ \\
\hline Med. Utilizados en el Diagnóstico & Medio Contraste No & $1(2 \%)$ \\
\hline Total Med. Utilizados en el & lónico lonversol & $1(2 \%)$ \\
\hline & & $1(2 \%)$ \\
\hline
\end{tabular}

$1(2 \%)$
$1(2 \%)$

$2(3 \%)$

Furosemida 2(3\%)

Hidroclorotiazida $\quad 1(2 \%)$

Nifedipino Ret $\quad 1(2 \%)$

$4(7 \%)$

$\begin{array}{ll}\text { Bupivacaína/Fentanilo } & 3(5 \%) \\ \text { Fenitoina } & 2(3 \%) \\ \text { Morfan } & 1(2 \%)\end{array}$

Morfina $\quad 1(2 \%)$

Petidina $\quad 1(2 \%)$

Tramadol

$1(2 \%)$

$8(14 \%)$

Frecuencia de Incidentes

$1(2 \%)$

$1(2 \%)$

Calcio

Enoxiparina $\quad 2(3 \%)$

Heparina 3(5\%) $1(19 \%)$ $(2 \%)$ $(3 \%)$

Levofloxacino $\quad 1(2 \%)$

Nitrofurantoína $\quad 1(2 \%)$

Benzilpenicilina $\quad 1(2 \%)$

Vancomicina $2(3 \%)$ 


\begin{tabular}{lr}
\hline Sin Información & Sin Información \\
\hline Total Sin Información & $3(5 \%)$ \\
N ${ }^{\circ}$ Casos Totales & $3(5 \%)$ \\
\hline
\end{tabular}

El $47 \%$ de los reportes de EM se iniciaron en el proceso de administración, y un 27\% en el proceso de dispensación (Figura 1).

Figura 1. Distribución de Errores de Medicación según el Proceso

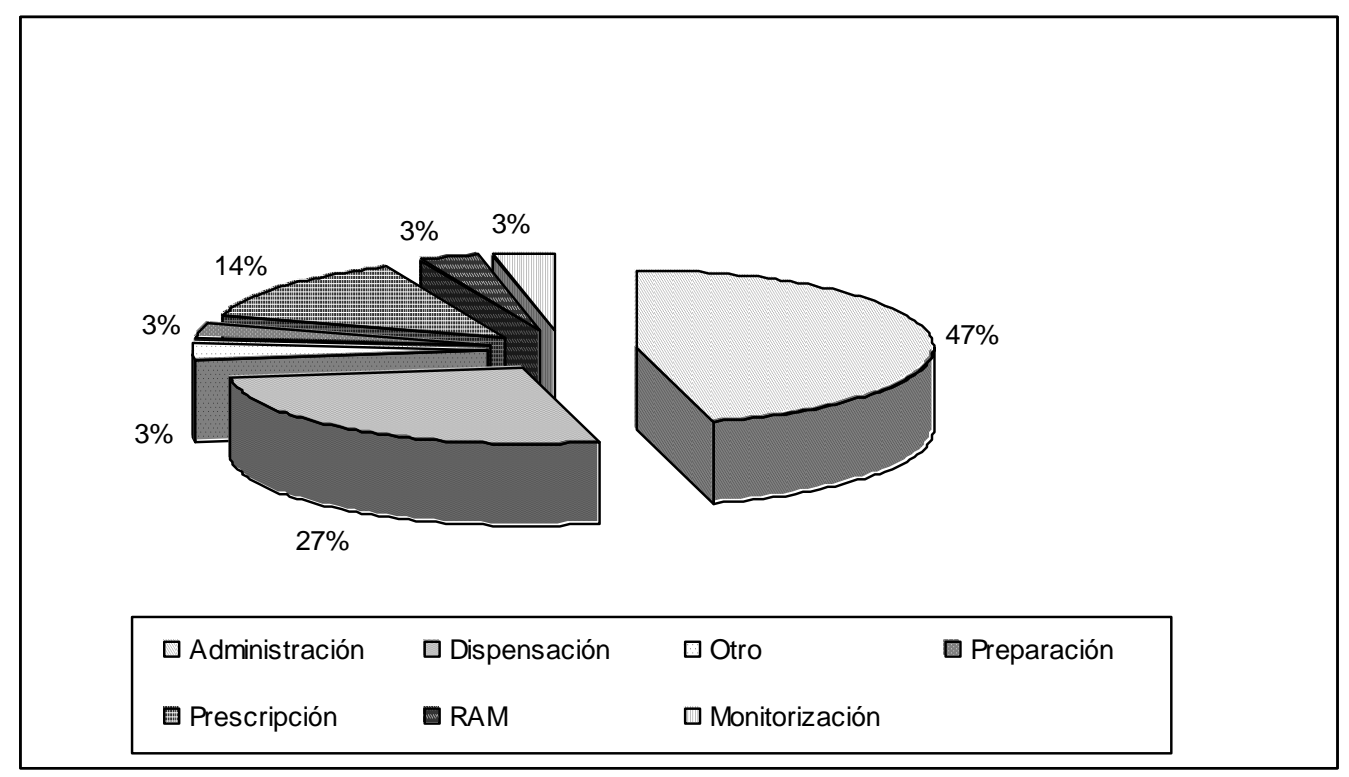

De los 59 reportes de EM, el $69 \%$ de ellos ${ }^{(41)}$ llegó al paciente, de los cuales el $34 \%$ necesitó monitorización, y el $39 \%$ necesitó que se adoptara más de una acción. Si bien no hubo muertes atribuibles a los EM reportados, el $47 \%$ se clasificó como tipo D, y el $97 \%$ de los EM reportados pudieron haber sido evitados (Tabla 4).

Tabla 4.- Gravedad y Grado de Prevención de los Errores de Medicación

\section{Gravedad}

\begin{tabular}{lr}
\hline A: Circunstancias o incidentes con capacidad de error & $8(14 \%)$ \\
B: Error se produjo pero no llegó al paciente & $17(28 \%)$ \\
C: Error alcanzó al paciente pero no causó daño & $6(10 \%)$ \\
D: Error alcanzó al paciente pero no causó daño, pero precisó de & \\
monitorización y/o intervención para comprobar que no hubo daño & $28(47 \%)$ \\
E: Error contribuyó o causó daño temporal al paciente y precisó & \\
intervención & $0 \%$ \\
F: Error contribuyó o causó daño temporal al paciente y precisó o & $0 \%$ \\
prolongó la hospitalización. & $0 \%$ \\
G: Error contribuyó o causó daño permanente al paciente. & \\
H: Error comprometió la vida del paciente y se precisó intervención & $0 \%$ \\
para mantener su vida. & $0 \%$ \\
lerror contribuyó o causó la muerte del paciente &
\end{tabular}


Grado de Prevención

\begin{tabular}{lr}
\hline No Prevenible & $1(2 \%)$ \\
Prevenible & $58(98 \%)$
\end{tabular}

En 46 de los 59 reportes de E.M. se pudo establecer el tipo de E.M. ocurrido en cada uno de los procesos (Tabla 5).

Tabla 5 Descripción de los Tipos de E.M. reportados

\begin{tabular}{|c|c|c|}
\hline ipo de EM & Descripción & Total \\
\hline & Paciente Equivocado & $4(18.2 \%)$ \\
\hline & Horario Equivocado & $3(13.6 \%)$ \\
\hline & Medicamento Equivocado & $6(27.3 \%)$ \\
\hline \multirow[t]{4}{*}{ Administración } & Dosis Equivocada & $3(13.6 \%)$ \\
\hline & Vía Equivocada & $1(4.5 \%)$ \\
\hline & Velocidad de Infusión & \\
\hline & Equivocada & $5(22.7 \%)$ \\
\hline \multirow[t]{3}{*}{ Total Administraciór } & & $22(47 \%)$ \\
\hline & Dispensación Tar & $1(10.0 \%)$ \\
\hline & mento Equivocado & $4(40.0 \%)$ \\
\hline \multirow[t]{3}{*}{ Dispensación } & Falta & $1(10.0 \%)$ \\
\hline & & $2(20.0 \%)$ \\
\hline & Mal Rotulado & $1(10.0 \%)$ \\
\hline \multicolumn{2}{|c|}{ Total E. Dispensación } & $10(22 \%)$ \\
\hline & Perdida & \\
\hline \multirow[t]{2}{*}{$\begin{array}{l}\text { Banales } \\
\text { Total E. Banales }\end{array}$} & Matraz & $\begin{array}{l}1(100 \%) \\
1(2.2)\end{array}$ \\
\hline & $\begin{array}{l}\text { Reconstitución Errónea } \\
\text { Velocidad de Infusión }\end{array}$ & $2(28.6 \%)$ \\
\hline \multirow[t]{2}{*}{ Preparación } & $\begin{array}{l}\text { Equivocada } \\
\text { Forma Farmacéutica }\end{array}$ & $5(71.4 \%)$ \\
\hline & Equivocada & $1(10.0 \%)$ \\
\hline Total Dispensación & & $10(22 \%)$ \\
\hline $\begin{array}{l}\text { Banal } \\
\text { Total Banal }\end{array}$ & Matr & $\begin{array}{r}1(100.0 \%) \\
1(2.2 \%)\end{array}$ \\
\hline Preparación & Reconstitución Errónea & $2(100.0 \%)$ \\
\hline \multirow[t]{2}{*}{ Total Preparación } & & $2(4.4 \%)$ \\
\hline & Letra & $1(14.3 \%)$ \\
\hline \multirow{3}{*}{ Prescripción } & No h & $3(42.9 \%)$ \\
\hline & Orde & $1(14.3 \%)$ \\
\hline & Dosis Equivocad & $2(28.6 \%)$ \\
\hline \multirow{4}{*}{$\begin{array}{l}\text { Total Prescripción } \\
\text { Reacción Adversa } \\
\text { a Medicamento } \\
\text { Total RAM }\end{array}$} & & $7(15.2 \%)$ \\
\hline & Rus & $1(50.0 \%)$ \\
\hline & Mal & $\begin{array}{r}1(50.0 \%) \\
2(4.4 \%)\end{array}$ \\
\hline & Medi & $1(50.0 \%)$ \\
\hline \multirow[t]{2}{*}{ Monitorización } & & \\
\hline & Parámetro de Laboratorio & $1(50.0 \%)$ \\
\hline \multirow{2}{*}{\multicolumn{2}{|c|}{$\begin{array}{l}\text { Total Monitorización } \\
\text { Total general }\end{array}$}} & $\%)$ \\
\hline & & \\
\hline
\end{tabular}


En el proceso de prescripción, el origen del error fue debido a que no había una receta $(42.9 \%)$ o bien porque la dosis prescrita no era la correcta (28.6\%). La entrega de un medicamento erróneo, fue el que originó el $40 \%$ de los E.M ocurridos en el proceso de dispensación. El $35.3 \%$ de los E.M. acontecidos en el proceso de administración se debió a la administración de un medicamento que no correspondía.

\section{DISCUSIÓN}

Este trabajo es de los pocos realizados en nuestro país que describen la experiencia acerca de los EM.

La tasa de reportes de errores en medicación en nuestro hospital fueron inferiores a las reportadas en estudios internacionales ${ }^{(17)}$ ya que el sistema llevaba poco tiempo de implementación, por lo cual es poco conocido. Otros motivos pueden ser la reticencia a reportar por parte del personal de salud involucrado en el EM por las posibles sanciones que puedan recibir. Entre otros motivos que han sido descritos en literatura como barreras para el reporte de EM están la falta de retroalimentación de las iniciativas tomadas posterior al reporte, falta de información para realizar un reporte y la dificultad para acceder a formularios de reportes ${ }^{(18)}$.

Llama la atención el alto número de EM que llegaron al paciente, valor cercano al $70 \%$, cifra superior a lo reportado en literatura ${ }^{(19)}$ y sea probablemente este motivo por el cual se hayan reportado.

Los procesos de utilización de medicamentos en los cuales se originaron los reportes de EM, son similares a otras experiencias publicadas ${ }^{(20)}$. Durante el análisis se pudo apreciar que en su mayor parte los EM reportados ocurrieron debido a diversas fallas dentro del sistema de uso de los medicamentos ${ }^{(21)}$. Se ha estimado que alrededor del $50 \%$ de los EM, se deben a omisión en procedimientos y la falta de automatización en el sistema de uso de medicamentos ${ }^{22)}$. Entre otras causas de los EM están: el desconocimiento de un medicamento al prescribirlo o administrarlo, dificultad para el acceso de información de medicamentos o protocolos, falta de comunicación entre el equipo de salud, etc. ${ }^{(23)}$

El horario en que ocurrieron los EM (datos no mostrados) en nuestro hospital, se concentró fundamentalmente en el horario diurno, posiblemente por la carga asistencial que se concentra en ese horario (toma de exámenes, mayor número de administración de medicamentos); es probable que la notificación en este horario sea mayor, por el control dado por la dotación completa de personal, no así durante el horario nocturno. Estos resultados son similares a otros estudios en que los EM se presentan entre las $12 \mathrm{pm}$ y $4 \mathrm{pm}{ }^{(24)}$, pero también porque en esos horarios existe mayor control, ya que existe dotación de personal completa, lo que no ocurre durante el horario nocturno.

La debilidad del presente trabajo, proviene de la calidad de la información entregada en los reportes de incidentes, ya que en muchas oportunidades no se tuvieron los datos completos de los pacientes, no se identificó con precisión la hora del incidente, ni las medidas que se tomaron para revertir el evento adverso, como también la descripción del cómo ocurrió el evento en sí. De esto se concluye que sobre la utilidad de los reportes de incidentes solamente serán útiles si ellos contienen toda la información con la se pueda identificar las causas de los EM y tomar medidas 
correctivas ${ }^{(25)}$. Los reportes de EM, detectan aquellos de alta significación clínica y tienen una baja tasa de falsos positivos ${ }^{(26)}$.

La situación descrita en el presente trabajo puede haber cambiado en la actualidad, ya que el hospital ha hecho difusión de las normas de buena práctica en lo que se refiere a administración de medicamentos, las que fueron elaboradas por una comisión mixta en la que participaron enfermeras de los diferentes servicios, matronas y químicos farmacéuticos. Con esto se espera que haya una homogeneidad en el proceso, ayudando a una mejora de la práctica asistencial.

\section{CONCLUSIONES}

Los EM son una realidad transversal para todos los servicios de nuestro centro asistencial. El reporte de EM, son un método útil para la detección del origen de los EM y para aquellos EM que tienen una alta significancia clínica para el paciente, sin embargo son sub-notificados.

Los EM están incluidos dentro del componente de la Seguridad en la Atención del Paciente que debe ser cumplido por todos los prestadores de salud para lograr la acreditación, la cual se puede lograr con la implementación de estándares de atención (normas de administración, guías de práctica clínica), protocolos de trabajo para el uso de medicamentos con alta probabilidad de EM (uso de insulinas, hipoglicemiantes orales, heparinas, etc), e implementando sistemas informáticos de prescripción y dispensación. Es importante considerar que los EM no sólo son debido al error humano, sino que también se ha de contemplar la mala planificación de los sistemas de trabajo y sistemas de distribución de medicamentos al paciente. Además se debe tener en cuenta que en los EM no sólo está involucrado el personal sanitario (médicos, farmacéuticos y enfermeras), sino también la industria farmacéutica e incluso las autoridades sanitarias y el propio paciente.

\section{REFERENCIAS}

1. Linda T. Kohn, JMC, And, Molla S. Donaldson, E. To Err Is Human: Building A Safer Health System. America, CoQoHCi, editor. Washington, D.C.: Nationale Academy Press; 1999.

2. Bates Dw., CD, Laird N., Petersen La., Small Sd., Servi D., Laffel G., Sweitzer Bj., Shea Bf., Hallisey R., Vander Vliet M., Nemeskal R., Leape LI. Incidence of Adverse Drug Events and Potencial Adverse Drug Events. JAMA. 1995;274:29-34. 3. Forster Aj., AT, Clark Hd., AI Saied G., Code Cc., Caughey Sc., Baker K., Watters J., Worthington J., Van Walraven C. Ottawa Hospital Patient Safety Study: incidence and timing of adverse eventes in patients admitedd to Canadian teaching hospital. CMAJ. 2004;170(8):1235-40.

4. $\quad$ Bates Dw., ME, Cullen Dj., Burdickl., Williams L., Laird N., Petersen La., Small Sd., Sweitzer Bj., Vander Vliet M., Leape LI. Patient Risk Factors for Adverse Drug Events in Hospitalized Patient. Arch Intern Med. 1999;159:2553-60.

5. Classen Dc., PS, Evans Sr.,Lloyd Jf.,Burke Jp Adverse Drug Events in Hospitalized Patient: Excess Length of Stay, Extra Costs, and Attributable Mortality. JAMA. 1997;277:301-6.

6. Mongeau, C. Voices from Colorado. Nursing. 1998;28(6):48-9.

7. Kaushal R, SK, Bates Dw. Effects of computerized physician order entry and clinical decision support systems on medication safety: a systematic review. Arch Intern Med. 2003;163(12):1409-16. 
8. Greengold NI, SR, Schneider P, Flynn E, Elashoff J, Hoying Cl, Barker K, Bolton Lb. The impact of dedicated medication nurses on the medication administration error rate: a randomized controlled trial. Arch Intern Med 2003;163(19):2359-67.

9. Valenzuela Gámez J. C. , LGaM, Pedraza Cezón L. A., Andrés Navarro N., González Manzanares J. L. Proyecto de implantación del sistema de distribución de medicamentos en dosis unitarias en una unidad de cuidados intensivos. Farm Hosp. 2005;29(5): 318-22.

10. Bryony D., SM, Vicent C., Barber N. Causes of prescribing error $\mathrm{n}$ hospital inpatients: a prospective study. The Lancet. 2002;359:1373-8.

11. Dean B., BN. Validity and Reliability of Observational Methods for Studying Medication Administration Errors. Am J Health-Syst Pharm. 2001;58(1):54-9.

12. Egger T, DH, Ahne G, Runge U, Neubert a, Criegee-Rieck M, Gassmann Kg, Brune $\mathrm{K}$. Identification of adverse drug reactions in geriatric inpatients using a computerised drug database. Drugs Aging. 2003;20(10):769-76.

13. Miller M.R., CJS, Lehmann C.U. Computer based medication error reporting: insights and implications. Qual Saf Health Care. 2006(15):208-13.

14. System, DOMaHI, Organization, WH. International Statistical Classification of Diseases and Related Health Problems Switzerland 2003 [Última Actualización 2003; Último Ingreso 200615 Enero]; 10th:[Disponible en:

http://www3.who.int/icd/vol1htm2003/fr-icd.htm.

15. Methodology, WCCFDS. ATC/DDD Index 2006. Oslo2006 [Última Actualización 2006-01-11; Último Ingreso 200616 Enero]; Disponible en: http://www.whocc.no/atcddd/.

16. Nccmerp, NCCFMERaP. Taxonomy of Medication Errors. 2008 [Último Ingreso Agosto]; Disponible en: http://www.NCCMERP.org.

17. Johnston Pe, FD, Byrne Dw, Murff Hj, Lee B, Stiles Ra, Speroff T. Assessment of adverse drug events among patients in a tertiary care medical center. Am J Health Syst Pharm. 2006;63(22):2218-27.

18. Handler Sm, PS, Olshansky Ef, Studenski Sa, Nace Da, Fridsma Db, Hanlon Jt. Identifying modifiable barriers to medication error reporting in the nursing home setting. J Am Med Dir Assoc 2007;8(9):568-74.

19. Corral Baena S, GaM, Beltrán García M, Salas Turrens J. Use of MBDS as a tool for the detection of drug-related adverse events. Farm Hosp. 2004;28(4):258-65.

20. Lapointe Nm, JJ. Medication errors in hospitalized cardiovascular patients. Arch Intern Med. 2003;163:1461-6.

21. Kopp Bj, EB, Allen Me, Theodorou Aa, Priestley G. Medication errors and adverse drug events in an intensive care unit: direct observation approach for detection. Crit Care Med. 2006;34(2):415-25.

22. Lisby M., NLP, Mainz J. Errors in the medication process: frequency, type, and potential. International Journal for Quality in Health Care. 2005;17( 1):15-22

23. Nichols P., CT, Craib I.A., Hopkins P., Bruce D.G. Learning from error: identifying contributory causes of medication errors in an Australian hospital. MJA. 2008;188:276-9.

24. Lesar Ts., BL, Delcoure K., Parmalee Jc., Masta-Gornic V., Pohl H. Medication Prescribing Error in a Teaching Hospital. JAMA. 1990;263(17):2329-34.

25. Leape, L. Reporting of Adverse Events. N Engl J Med. 2002;347(20):1633-8. 26. Flynn Ea, BK, Pepper Ga, Bates Dw, Mikeal RI. Comparison of methods for detecting medication errors in 36 hospitals and skilled-nursing facilities. Am J Health Syst Pharm. 2002;59(5):436-46. 
๑ COPYRIGHT Servicio de Publicaciones - Universidad de Murcia 